INGENIERÍA DE TRANSPORTE

\title{
Cómo modelar la elección del modo caminata con base en datos de encuestas domiciliarias
}

TRANSPORT ENGINEERING

\section{Modeling the choice of walking based on household survey data}

\author{
Luis Márquez*§, Domingo Dueñas*, Luis F. Macea** \\ * Programa de Ingeniería de Transporte y Vias, Grupo de Investigación y Desarrollo en \\ Planeación y Operación del Transporte - GIDPOT, Universidad Pedagógica y Tecnológica de \\ Colombia, Tunja, Colombia. \\ ** Departamento de Ingeniería Civil y Ambiental, Universidad del Norte, Barranquilla, Colombia \\ §luis.marquez@uptc.edu.co,doduenasr@yahoo.com,lmacea@outlook.com
}

Recibido: 15 de Agosto de 2013 - Aceptado: 15 de Octubre de 2014

\begin{abstract}
Resumen
Los estudios de planificación del transporte urbano adelantados en Colombia muy pocas veces incluyen la modelación de los viajes peatonales, en especial para establecer la probabilidad de caminar. Este artículo reporta los resultados de la investigación para analizar la elección del modo caminata de las personas en un contexto urbano con base en datos de encuestas domiciliarias. El objetivo principal de la investigación es demostrar cómo la información proveniente de encuestas domiciliarias sirve para modelar la probabilidad de caminar en una ciudad. Son utilizados datos de encuestas realizadas en dos ciudades colombianas (Barrancabermeja y Cajicá) con los cuales se estiman modelos de elección. Los resultados del estudio indican que la probabilidad de caminar disminuye a medida que aumentan los valores de atributos tales como tiempo de viaje, número de autos en la familia e ingreso personal, y aumenta cuando se trata de personas jóvenes y grupos familiares de mayor tamaño.
\end{abstract}

Palabras clave: Elección del modo caminata, encuestas a hogares, modelo Logit multinomial, preferencias reveladas, transporte no motorizado.

\begin{abstract}
In Colombia, urban transport planning studies do not always include the modeling of pedestrian travel, particularly to study the choice of walking. This paper reports the results of research to model the choice of walking in an urban context, based on household survey data. The purpose of this research is to demonstrate how household surveys can be used to study the choice of traveling by foot in a city. Data from surveys conducted in two Colombian cities (Barrancabermeja and Cajicá) are used. With the information available it was possible to estimate choice models of travel by foot, being also possible to identify the attributes that affect the decision to travel on foot. The study results indicate that the probability of walking decreases as travel time increases. Also, more cars and more income mean less chance of walking. Instead, the probability of walking increases for young people and family groups larger.
\end{abstract}

Key words: Choosing to travel by foot, household surveys, logit multinomial model, revealed preference, nonmotorised transport. 


\section{Introducción}

La modelación de los desplazamientos peatonales en el entorno urbano de las ciudades ha sido de gran interés para la comunidad científica mundial (Löhner, 2010); sin embargo, en el contexto de los estudios de movilidad urbana realizados en Colombia, basados normalmente en encuestas domiciliarias, el análisis de estos viajes no se aborda con el mismo rigor aplicado en el estudio de los viajes motorizados.

Sobre la base de la literatura existente es razonable suponer que la elección del patrón de viajes personales depende de las necesidades de participar en el sistema de actividades y puede ser estudiado como una función de ciertos atributos del medio ambiente urbano, familiar e individual, limitado por un conjunto de opciones disponibles asociadas particularmente con las características del sistema de transporte (Pitombo et al., 2011). Investigaciones llevadas a cabo en países desarrollados han encontrado que una alta densidad urbana propicia el uso de modos de transporte no motorizados, como caminar y usar bicicleta, bajo el supuesto que las distancias entre destinos son cortas (Cervero \& Kockelman, 1997; Ewing et al., 2003); otros atributos, que son más difíciles de medir pero que se relacionan también con la influencia del medio ambiente en la caminata y que han sido estudiados desde perspectivas distintas a los análisis de movilidad propiamente dichos, son el nivel de percepción de la estética del medio ambiente y las zonas verdes (Cerin et al., 2009).

En el ámbito familiar cobran importancia atributos como el ingreso del núcleo familiar (Pitombo et al., 2011) la posición del individuo en la familia (Schwanen et al., 2002) y otros ampliamente tratados en los textos de planificación de transporte, como el tamaño del hogar y la tasa de motorización (Bohnet \& Gertz, 2008), que son utilizados con relativo éxito en la modelación de generación de viajes personales (Ortúzar \& Willumsen, 2011), en el marco de la estructura del modelo clásico de los cuatro pasos. Con relación a los atributos individuales se ha encontrado que la frecuencia de la caminata está relacionada con el nivel educativo, los ingresos personales y la intensidad de la actividad física desarrollada por las personas durante su tiempo libre (Cerin et al., 2009), siendo altamente significativo el efecto de los ingresos personales.

En el marco de los estudios de movilidad urbana adelantados en Colombia, particularmente aquellos que se basan en encuestas domiciliarias, son medidos varios atributos del sistema de actividades, del sistema de transporte, del medio ambiente, de los hogares y de los individuos, y normalmente la medición de estos atributos se hace en forma homogénea, puesto que las técnicas utilizadas siguen los lineamientos del manual para estudios de origen y destino de transporte de pasajeros y mixto en áreas municipales distritales y metropolitanas, adoptado por el Ministerio de Transporte de Colombia (1998) mediante reglamentación. A pesar de que los datos recopilados en las encuestas domiciliarias permitirían abordar su análisis, los estudios de planificación de transporte urbano desarrollados en las ciudades colombianas rara vez involucran los viajes peatonales en las tareas de modelación, por tal razón se considera de especial interés evaluar cómo los datos provenientes de encuestas domiciliarias pueden ser utilizados en la construcción de modelos que permitan analizar, entre otros tópicos, la probabilidad de elegir el modo de viajar a pie.

Uno de los retos del análisis propuesto es encontrar una forma apropiada de incorporar la información estadística proveniente de estos estudios de preferencias reveladas para describir y modelar las variaciones de actitud de los individuos (Jakobsson et al., 2011; Johansson et al., 2006), incorporando además atributos del entorno del cual el individuo es parte (Dugundji et al., 2011). $\mathrm{Al}$ respecto, la técnica de regresión no lineal para estimar modelos de elección ha sido utilizada con éxito para analizar los factores que inciden en el uso de modos no motorizados como la bicicleta y la caminata, mediante la estimación de modelos que incluyen la pendiente predominante en la infraestructura, la densidad y uso de suelo de la zona, el estrato socioeconómico socio-económico y la tenencia de autos en el hogar, y algunas

\section{6}


características individuales como la edad (Cervero et al., 2009). En este enfoque de modelación, la decisión de viajar a pie ha sido tratada como una variable binaria, mediante el uso de modelos de tipo Logit que incluyen variables explicativas como duración del viaje, edad, sexo, ingreso personal y propiedad de automóviles, tal como se ve en un trabajo publicado recientemente, que se basa en una encuesta origen destino aplicada durante el año 2003 en Montreal (Manaugh \& ElGeneidy, 2011).

Manteniendo esta línea de análisis, en el presente artículo se reportan los resultados de la investigación para analizar la probabilidad de caminar de los individuos con base en datos de encuestas domiciliarias; se propone como hipótesis central tratar de demostrar, desde el punto de vista metodológico, funcional y de posibilidades de aplicación, cómo los datos provenientes de encuestas domiciliarias de preferencias reveladas, pueden servir para analizar los atributos que determinan la probabilidad de caminar de los individuos en un ámbito urbano. Para tal fin, son utilizados datos de encuestas domiciliarias provenientes de dos estudios, aplicados en ciudades de características distintas, en épocas diferentes, con objetivos heterogéneos y que convergen en la técnica aplicada para la obtención de las observaciones.

Dado que el enfoque propuesto prevé la utilización de información de encuestas domiciliarias, surgen algunas limitaciones que es justo reconocer. En primer lugar, aunque en la práctica de la modelación del transporte en Colombia, recientemente se ha abordado el problema de la modelación híbrida (ver por ejemplo Márquez et al., 2014), en este caso no es posible especificar variables latentes, tales como la seguridad y la conveniencia asociadas a la caminata, ya que las bases de datos de encuestas domiciliarias no cuentan con información que pudiese emplearse a manera de indicadores de las variables no observables.

Otra limitante a considerar es la dificultad de incluir variables asociadas con el entorno, que como es bien sabido, tienen que ver con la elección del modo caminata, tal como lo describen Cao et al. (2006), Handy et al. (2006), Mokhtarian \& Cao (2008), y Larrañaga et al. (2013). Ahora bien, la posibilidad de incluir este tipo de información implicaría la utilización de fuentes secundarias de datos, diferentes a las encuestas domiciliarias.

\section{Metodología}

\subsection{Encuesta domiciliaria}

Los datos utilizados provienen de unas encuestas domiciliarias de preferencias reveladas, aplicadas en los municipios de Barrancabermeja y Cajicá, en desarrollo de los estudios realizados para la re-estructuración del sistema de rutas de Barrancabermeja y para la formulación del plan de movilidad de Cajicá, en los años 2005 y 2010, respectivamente.

La información recopilada homogéneamente en ambos estudios, se relaciona con características básicas de la vivienda, datos de los residentes, relación de vehículos disponibles por hogar con sus características y diario de viajes. En este último caso, para un día hábil típico, se registró información referida a origen, destino, hora de inicio y terminación, costos, modo de transporte utilizado y motivo del viaje, entre otros aspectos que permiten caracterizar cada viaje realizado.

Barrancabermeja se dividió en 72 zonas de análisis de transporte, como se observa en la figura 1, y Cajicá se dividió en 44 zonas que incluyen el área rural del municipio, como se ve en la figura 2, donde además se observa que las zonas rurales periféricas son de tamaño considerablemente mayor al de las urbanas, localizadas en el centro del municipio.

En ambos casos se utilizó una técnica de muestreo por conglomerados, en dos etapas; en la primera etapa, para cada zona de análisis de transporte se seleccionó aleatoriamente un subconjunto de manzanas y en la segunda fase fue seleccionado al azar un subconjunto de viviendas. En los dos contextos estudiados se verificó que la muestra tomada no fuese inferior al $5 \%$ de las viviendas; en el caso de Barrancabermeja se conformó una muestra de 2,045 viviendas, y en Cajicá fueron 


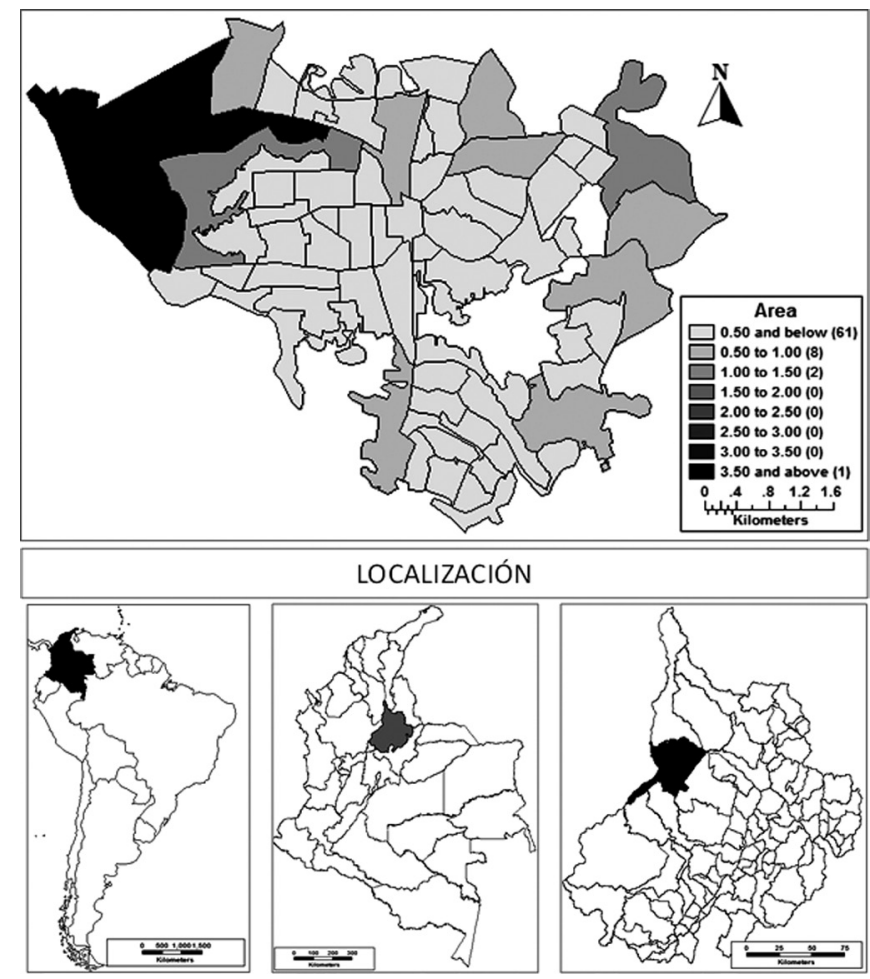

Figura 1. Zonificación de Barrancabermeja

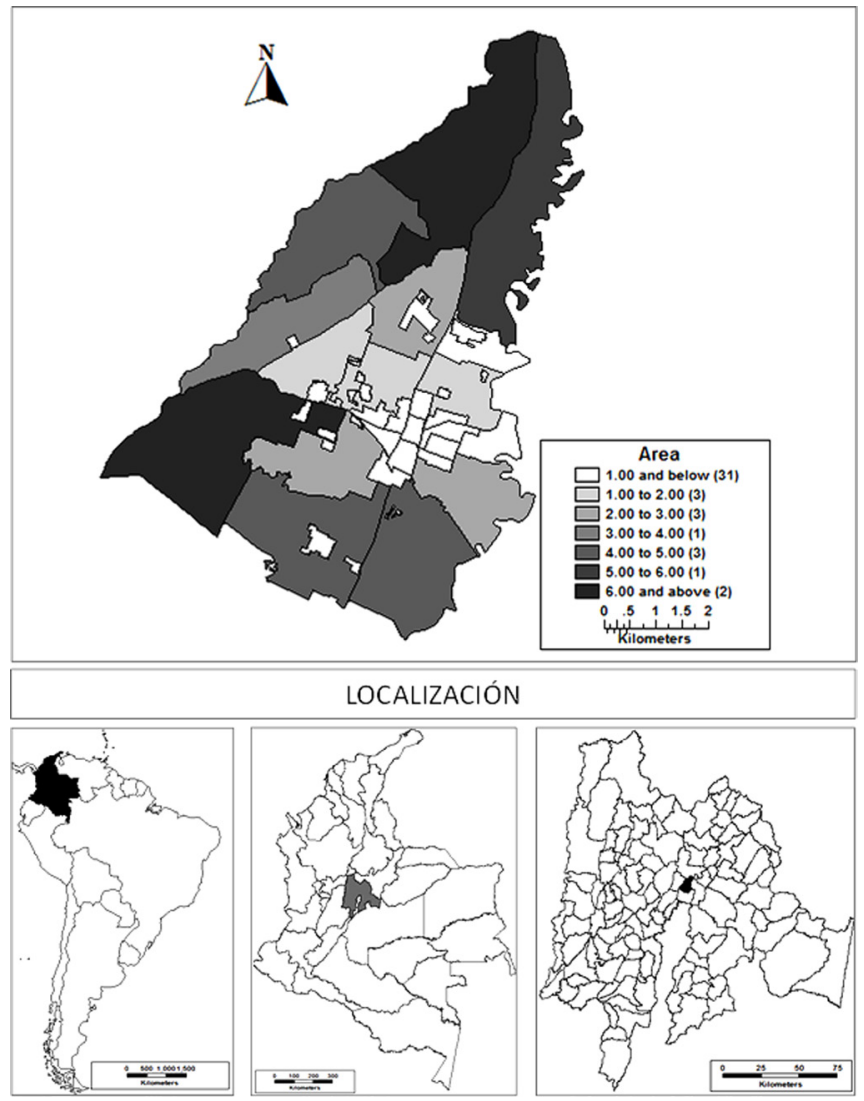

Figura 2. Zonificación de Cajicá 
visitadas 486 viviendas. Aunque la unidad de muestreo fue la vivienda, las observaciones se hicieron por hogar, tomando información a nivel de los individuos, tal como sexo, edad e ingresos personales.

\subsection{Caracterización de la muestra}

Las características generales de los dos municipios estudiados son diferentes. Para la época del estudio, Barrancabermeja tenía una población de 170,810 habitantes, localizados predominantemente en el perímetro urbano, con una densidad de 5,624 $\mathrm{hab} / \mathrm{km}^{2}$. Cajicá, en cambio, es un municipio más pequeño, con una población estimada para el año 2010 de 51,100 habitantes, distribuidos con escasa diferencia en la zona urbana $(54 \%)$ y rural (46\%). La densidad poblacional de Cajicá también es menor, ubicándose en $841 \mathrm{hab} / \mathrm{km}^{2}$. La baja densidad y la distribución espacial atípica de la población en Cajicá fueron las razones por las cuales se decidió aplicar el estudio tanto en el área urbana como rural, al mismo tiempo que las veredas se caracterizan por tener un buen de número de residencias de estrato socioeconómicos altos y por lo tanto su comportamiento es similar al urbano.

Se verificó la correspondencia de las muestras comparando las distribuciones muestrales de las principales variables demográficas en cada municipio con respecto a la distribución poblacional. Las variables con las que se hizo tal verificación fueron sexo, edad, tasa de motorización y nivel de estudios, encontrando en todos los casos una aceptable similitud de las distribuciones muestrales con las poblacionales.

\subsection{Modelación}

Los modos de transporte disponibles, el núcleo familiar, los individuos y los viajes, se procesaron en forma desagregada, obteniendo un registro para cada uno de los viajes observados en la muestra a nivel del individuo. En Barrancabermeja se conformó una base de datos con 13,554 registros y en Cajicá se logró consolidar un total de 3,660 registros. En cada caso se identificó si el viaje había sido hecho a pie o en cualquier otro modo de transporte, utilizando una variable binaria que tomó el valor de 1 para los viajes realizados a pie y 0 para los demás modos de transporte.

Los datos recopilados fueron objeto de un análisis estadístico descriptivo y con las principales variables explicativas se procedió a la estimación de modelos de elección de tipo Logit. Se descartó el uso de modelos lineales de probabilidad dado que se ha demostrado que no proporcionan una respuesta adecuada en la modelación de procesos de decisión dicotómica, a diferencia de las especificaciones no lineales que solucionan algunos de los problemas asociados (Alamilla \& Arauco, 2009).

En el modelo Logit multinomial, al suponer que los términos de error $\varepsilon_{\mathrm{ni}}$ distribuyen en forma idéntica e independiente Gumbel, la probabilidad de que un individuo $\mathrm{n}$ elija la alternativa $\mathrm{i}$, viene dada por:

$$
p_{n i}=\frac{e^{V_{n i}}}{\sum_{j} e^{V_{n j}}}
$$

Donde, $V_{n i}$ es la utilidad representativa o sistemática. En la mayor parte de los modelos de elección discreta que se emplean en la actualidad, se especifican funciones lineales y se considera que los parámetros $\beta$ son constantes para todos los individuos pero pueden variar entre las alternativas (Train, 2003), así:

$$
V_{n i}=\sum_{k} \beta_{k} \cdot X_{k n i}
$$

Para estimar el modelo Logit multinomial se debe observar una muestra de las elecciones de $N$ individuos, y sobre ella aplicar la técnica de estimación por máxima verosimilitud; en el caso de interés, las observaciones provienen de las bases de datos de encuestas domiciliarias, que se toman con técnicas de preferencias reveladas. La probabilidad de que un individuo elija la alternativa que efectivamente escogió está dada por:

$$
\prod_{i}\left(p_{n i}\right)^{Y_{n i}}
$$


Donde, $\mathrm{Y}_{\mathrm{ni}}$ toma el valor de 1 si el individuo n elije viajar a pie, y 0 en otro caso. Asumiendo que las decisiones de los individuos son independientes entre sí, la probabilidad que cada individuo en la muestra elija la alternativa que se observó está dada por:

$$
L(\beta)=\prod_{n=1}^{N} \prod_{i}\left(p_{n i}\right)^{Y_{n i}}
$$

Donde, $\beta$ es el vector que contiene los parámetros del modelo. Entonces, la función de logverosimilitud queda así:

$$
l(\beta)=\sum_{n=1}^{N} \sum_{i} Y_{n i} \cdot \ln \left(p_{n i}\right)
$$

El proceso de calibración consiste en la estimación de los coeficientes $\beta$, tomando como estimadores aquellos valores que maximicen la verosimilitud de la muestra utilizada (Train, 2003). Cuando se alcanza la convergencia y se obtienen los valores que maximizan la verosimilitud se debe cumplir que:

$$
\frac{\partial l(\beta)}{\partial \beta}=0
$$

Esto significa que los estimadores máximoverosímiles son entonces aquellos valores de $\beta$ que satisfacen las condiciones de primer orden.

La selección del mejor modelo se basa en el examen y comparación de algunos indicadores tales como la consistencia de signos, la significancia de los estimadores, medidas de bondad de ajuste mediante los índices de razón de verosimilitud $\rho$ y $\rho^{2}$ (Ortúzar \& Willumsen, 2011) y la aplicación del test de razón de verosimiltud para comparar modelos más complejos frente a otros más sencillos que pueden obtenerse mediante restricciones lineales (BenAkiva \& Lerman, 1985).

Una vez que el mejor modelo es elegido, además de su empleo para realizar pronósticos de elecciones en diferentes escenarios, puede aprovecharse para medir elasticidades con respecto a diferentes variables tales como tarifas y demás atributos (Train, 2003). Así, la variación porcentual en la probabilidad de utilizar una alternativa i con respecto a la variación porcentual de una variable determinada se calcula como:

$$
E_{i X_{n i}}=-\beta_{X}\left(1-p_{n i}\right) \cdot X_{i}
$$

Donde, $\beta_{X}$ es el coeficiente estimado de la variable de interés, pni es la probabilidad de que el individuo $\mathrm{n}$ decida escoger la alternativa $i$, y $X_{i}$ es el valor medio de la variable.

Las variables incluidas en los mejores modelos permitieron identificar los atributos que inciden en la probabilidad de caminar de los individuos; además, los coeficientes estimados para esos atributos se usaron para medir elasticidades con respecto a diferentes variables, entre ellas el tiempo de viaje. Las elasticidades, para cada contexto analizado, fueron calculadas a partir de las observaciones individuales de cada una de las dos muestras tomadas.

\section{Resultados y discusión}

En Barrancabermeja se encontró un total de 268,944 viajes diarios, de los cuales el $31 \%$, es decir 83,373 viajes diarios, se hizo a pie; en Cajicá, entre un total de 84,138 viajes diarios, se precisó que solamente el $8.23 \%$ se hace a pie, lo que corresponde a 6,925 viajes diarios. Estos resultados, tratados como la relación existente entre densidad poblacional y cantidad de viajes efectuados a pie, son consistentes con las deducciones de otros estudios (Cervero \& Kockelman, 1997; Ewing et al., 2003), en los cuales se ha encontrado que una mayor densidad poblacional favorece la elección de viajar a pie.

El análisis estadístico descriptivo de las variables utilizadas en la construcción de modelos se presenta en la tabla 1 . Se encontró que el tiempo medio de viaje es mayor en Cajicá, aproximadamente unos 9 minutos más, lo cual corresponde con la menor densidad de ese municipio en comparación con el caso de Barrancabermeja y en consecuencia se supone razonable; no obstante, los tiempos medios de 
Tabla 1. Estadísticas descriptivas

\begin{tabular}{|c|c|c|c|c|}
\hline \multirow{2}{*}{$\begin{array}{c}\text { Variables } \\
\text { Numéricas }\end{array}$} & \multicolumn{2}{|c|}{ Barrancabermeja } & \multicolumn{2}{|c|}{ Cajicá } \\
\hline & Media & Desviación & Media & Desviación \\
\hline \multicolumn{5}{|l|}{ Tiempo de viaje (min) } \\
\hline Todos los modos & 21.16 & 28.07 & 30.60 & 56.913 \\
\hline Apie & 19.82 & 23.51 & 20.11 & 24.72 \\
\hline Tamaño del hogar & 4.777 & 2.082 & 3.175 & 0.640 \\
\hline Número de vehiculos por hogar (incluye motos) & 0.946 & 0.893 & 0.858 & 0.538 \\
\hline Edad & 18.550 & 14.106 & 31.750 & 17.813 \\
\hline $\begin{array}{l}\text { Ingreso personal (salarios mínimos por } \\
\text { persona) }\end{array}$ & 0.228 & 0.621 & 1.264 & 1.488 \\
\hline Categóricas & $\%$ & & $\%$ & \\
\hline $\begin{array}{l}\text { Sexo } \\
\text { 1: Hombre } \\
\text { 0: Mujer }\end{array}$ & $\begin{array}{l}43.5 \% \\
56.5 \%\end{array}$ & & $\begin{array}{l}46.6 \\
53.4\end{array}$ & \\
\hline $\begin{array}{l}\text { Estrato socioeconómico } \\
\qquad \begin{array}{c}1-2 \\
3-4 \\
5-6\end{array}\end{array}$ & $\begin{array}{c}50.3 \% \\
46.9 \% \\
2.8 \%\end{array}$ & & $\begin{array}{l}25.2 \% \\
71.6 \% \\
3.2 \%\end{array}$ & \\
\hline
\end{tabular}

caminata son similares en ambos contextos, con un valor que ronda los $20 \mathrm{~min}$. Aunque hubiese sido preferible estudiar la impedancia de los viajes en función de la longitud, en lugar del tiempo de viaje, con el fin de evitar las distorsiones producidas por modos de transporte con diferentes velocidades, esto no fue posible debido a que las distancias de los viajes observados en la encuesta se calcularon en una fase post-proceso aplicando algoritmos de rutas mínimas entre zonas, lo que impidió conocer la verdadera longitud de los viajes intrazonales, muchos de ellos realizados a pie.

El tamaño del hogar resultó significativamente mayor en Barrancabermeja, lo cual es coherente con un menor estrato socioeconómico. Se encontró que la tenencia de vehículos en los dos municipios no corresponde con los estratos socioeconómicos predominantes ni con los niveles de ingresos estimados por hogar, ya que Cajicá, a pesar de tener un ingreso mayor, reportó una tasa de motorización menor, debido a la elevada presencia demotocicletas en el municipio de Barrancabermeja.

Con respecto al comportamiento del atributo sexo, en ambos casos se encontró mayor número de mujeres, en proporciones razonables con respecto a los indicadores censales de cada municipio. Al contrastar la edad promedio de los habitantes en los dos municipios, se encontró que Cajicá es un municipio menos joven que Barrancabermeja, situación que concuerda con las diferencias en las cuotas de mercado para el modo de viajar a pie, bajo el entendido que a menor edad mayor disposición a caminar.

La tabla 2 presenta los parámetros estimados y las medidas de ajuste para el mejor modelo de tipo Logit que se logró obtener en cada caso. Los parámetros estimados permitieron establecer que, comparativamente con los demás modos de transporte disponibles en cada contexto de elección, la caminata en Cajicá tiene una menor utilidad, en virtud del menor valor estimado para el intercepto, lo cual es consistente con el reparto modal obtenido en las encuestas de preferencias reveladas. Se encontró que la probabilidad de caminar en los dos contextos estudiados disminuye en la medida que aumentan los valores de los atributos tiempo de viaje, número de autos, estrato socioeconómico, edad e ingreso personal; al respecto, ninguna de las especificaciones probadas para el caso de Cajicá permitió obtener un coeficiente significativo para el ingreso personal, debido posiblemente a una menor variación de esta variable con respecto al caso de Barrancabermeja, sin embargo el signo 
Tabla 2. Modelos estimados

\begin{tabular}{ccc}
\hline Descripción & Barrancabermeja & Cajicá \\
\hline Intercepto & $-0.466(-6.32)$ & $-3.7784(-8.12)$ \\
Tiempo de viaje & $-0.008(-6.11)$ & $-0.003(-3.20)$ \\
Tamaño del hogar & $0.248(7.63)$ & $0.781(8.13)$ \\
Número de autos & $-0.622(-22.17)$ & $-0.715(-5.76)$ \\
Estrato socioeconómico & & \\
1: Medio-Alto (Estratos 3, 4 y 5) & $-0.149(-4.32)$ & $-0.186(-2.04)$ \\
0: Bajo (Estratos 1 y 2) & & \\
Sexo (1: Hombre, 0: Mujer) & $0.061(1.96)$ & $0.092(1.94)$ \\
Edad & $-0.214(-1.97)$ & $-0.207(-1.67)$ \\
Ingreso personal & $-0.263(-6.49)$ & $-1,040.11$ \\
Log-verosimilitud inicial & $-8,414.09$ & -995.30 \\
Log-verosimilitud final & $-7,834.02$ & 89.62 \\
Razón de Log-verosimilitud & $1,160.14$ & 0.046 \\
$R^{2}$ (McFadden) & 0.070 & 7 \\
Parámetros estimados & 8 & 3,659 \\
Observaciones & 13,551 &
\end{tabular}

obtenido para esta variable fue siempre negativo, pero nunca con niveles de confianza superiores al $50 \%$.

Se pudo comprobar con una confianza superior al $99 \%$ que individuos pertenecientes a hogares de mayor tamaño tienen una mayor probabilidad de caminar. Este resultado puede ser de particular interés en la priorización de proyectos de peatonalización o construcción de andenes, ya que ceteris paribus este tipo de iniciativas debería considerar en primer lugar aquellos sectores con presencia de hogares de mayor tamaño, cuyos integrantes están más dispuestos a caminar.

Se determinó también que individuos más jóvenes tienen mayor probabilidad de caminar, situación que puede estar asociada con las actividades de estudio y con un menor nivel de ingresos personales. Con respecto al sexo se comprobó que los hombres tienen mayor probabilidad de caminar que las mujeres y aunque en el caso de Cajicá, la significancia del coeficiente que acompaña al sexo resultó menor al $95 \%$, se obtuvo el mismo signo que en el modelo estimado para Barrancabermeja, siendo consistente con otras publicaciones. Estos resultados difieren parcialmente de los hallazgos de Kitchen et al. (2011), quienes en el contexto de los viajes al trabajo en Canadá y desde una perspectiva epidemiológica, sobre la base de la aplicación de encuestas de salud, encontraron que las mujeres y los más jóvenes tenían una mayor probabilidad de caminar en viajes de menor duración pero menor probabilidad de caminar en viajes de más alta duración. En la modelación que se realizó en la presente investigación, la interacción de estas variables no resultó significativa en ninguna de las especificaciones probadas.

Esconveniente resaltarquelos modelos presentados no consideran ninguna variable asociada con el entorno donde se realizan los viajes, ya que las bases de datos provenientes de las encuestas domiciliarias normalmente no almacenan este tipo de información. Claramente esta es una limitación del enfoque propuesto ya que si las variables demográficas estuviesen correlacionadas con las variables de entorno (que no son digitadas en las bases de datos de encuestas domiciliarias), se habrían estimado entonces parámetros sesgados.

La estimación de modelos consideró varias especificaciones, incluso aquellas donde los parámetros de los atributos del modo o del viaje están en interacción con características individuales observadas tales como edad, 
Tabla 3. Elasticidades directas en los modelos

\begin{tabular}{cccc}
\hline \multicolumn{4}{c}{ Elasticidad para valores Medios } \\
\hline Descripción & Valor & Barrancabermeja & Cajicá \\
Tiempo de viaje (min) & 20 & & \\
Tamaño del hogar & 4 & & \\
Número de autos & 0 & & -0.0598 \\
Estrato socioeconómico & 3 & -0.1585 & \\
Sexo & 1 & & \\
Edad & 20 & & Cajicá \\
Ingreso personal (salarios) & 0,5 & & -0.0897 \\
\hline \multicolumn{5}{c}{ Elasticidades para otros valores } \\
\hline Descripción & Valor & Barrancabermeja & -0.1197 \\
& 30 & -0.2379 & -0.1496 \\
Tiempo de viaje (min) & 40 & -0.3175 & \\
& 50 & -0.3971 & \\
Ingreso personal (salarios) & 2 & -0.1587 & \\
& 3 & -0.1590 & \\
\hline
\end{tabular}

género e ingreso personal. Aunque este tipo de especificaciones permitiría recoger la variación sistemática de los gustos, ninguna de las especificaciones probadas resultó significativa. De otra parte, el alcance del trabajo no consideró la especificación de modelos de parámetros aleatorios, que como es bien sabido, siguen siendo poco utilizados en contextos prácticos.

Aplicando la Ec. (7) se determinó la elasticidad directa entre el tiempo de viaje y la probabilidad de caminar, inicialmente para los valores medios de los atributos y luego para distintas categorías de tiempo de viaje e ingreso personal, encontrando los valores que se muestran en la tabla 3 . Estos valores representan la variación de la probabilidad de caminar cuando se produce un cambio en el tiempo de viaje; las elasticidades directas tienen signo negativo indicando que ante aumentos de tiempos, la probabilidad de caminar disminuye.

La primera parte de la tabla 3 exhibe las elasticidades para los valores medios de las variables incluidas en el modelo, mientras que en la segunda parte se muestran las elasticidades obtenidas a partir de la variación de algún atributo, manteniendo todo lo demás constante. Se encontró que los habitantes del municipio de Barrancabermeja son más sensibles frente a cambios en los tiempos de viaje, en comparación con los habitantes de Cajicá, cuya elasticidad en términos absolutos corresponde al 38 $\%$ de la obtenida en Barrancabermeja. Así mismo, se halló que la elasticidad de la probabilidad de caminar con respecto al tiempo de viaje va aumentando en la medida que se tienen tiempos de viaje mayores, comportamiento que es consistente con análisis realizados para los viajes motorizados, donde la elasticidad es mayor en la medida que se consideran desplazamientos más largos (Litman, 2007).

\section{Conclusiones}

El trabajo realizado resalta la importancia de modelar los atributos que determinan la probabilidad de caminar por parte de los residentes en una ciudad, como herramienta de análisis integral en el marco de los procesos de planificación del transporte urbano, a fin de precisar las políticas y acciones conducentes a satisfacer las necesidades de desplazamiento en las ciudades.

Se ha desarrollado y probado un enfoque metodológico para el análisis estadístico descriptivo de los datos recopilados en encuestas 
domiciliarias y para el ajuste de modelos no lineales, encontrando que el modelo Logit es el más adecuado para los datos modelados, y que la búsqueda de los parámetros se puede realizar en forma sencilla y eficiente utilizando herramientas computacionales básicas.

En cuanto a los parámetros que explican en mejor forma la elección del modo caminata, son identificados los siguientes: tiempo de viaje, tamaño del grupo familiar, número de autos de la familia, sexo, edad e ingreso personal. Se manifiesta claramente que a medida que aumenta el tiempo de viaje se disminuye la probabilidad de caminar, así mismo, las personas pertenecientes a hogares de mayor tamaño y los jóvenes tienen una mayor disposición a desplazarse a pie.

Se propone la realización de trabajos futuros en los que se evalúen ciudades de diferentes características, con el fin de estudiar patrones de comportamiento de viajes a pie que ayuden a perfeccionar la toma de decisiones en materia de infraestructura y estrategias de gestión que mejoren la disposición a caminar de los habitantes.

\section{Referencias bibliográficas}

Alamilla-López, N. E., \& Arauco, S. (2009). Limitaciones del modelo lineal de probabilidad y alternativas de modelación microeconométrica. Temas de Ciencia y Tecnología 13 (39), 3-12.

Ben-Akiva, M. \& Lerman, S.R. (1985). Discrete Choice Analysis. Theory and Application to Travel Demand. The MIT Press. Cambridge, Massachusetts.

Bohnet, M., \& Gertz,C. (2008). Mode specific accessibility and car ownership. Technische Universität München, Institute for Transportation (Hrsg.). In: Proceedings of MOBIL. TUM 2008-International Conference on Mobility \& Transport, 08-09 April, Munich, Germany.

Cao, X., Handy, S. \& Mokhtarian, P. (2006) The Influences of the Built Environment and Residential Self-Selection on Pedestrian Behavior. Tx. Transportation 33(1), p.1-20.
Cerin, E., Leslie, E., \& Owen, N. (2009). Explaining socio-economic status differences in walking for transport: An ecological analysis of individual, social and environmental factors. Social Science \& Medicine 68, 1013-1020.

Cervero, R., \& Kockelman, K. (1997). Travel demand and the 3Ds: Density, Diversity, and Design. Transportation Research Part D 2, 199219.

Cervero, R., Sarmiento, O. L., Jacoby, E.; Gómez, L. F., \& Neiman, A. (2009). Influences of Built Environments on Walking and Cycling: Lessons from Bogotá. International Journal of Sustainable Transportation 3 (4), 203-226.

Dugundji, E. R., Páez, A., Arentze, T. A., \& Walker, J. L. (2011). Transportation and social interactions. Transportation Research Part A 45, 239-247.

Ewing, R., Schmid, T., Killingsworth, R., Zlot, A., \& Raudenbush, S. (2003). Relationship between urban sprawl and physical activity, obesity and morbidity. American Journal of Health Promotion $18,47-57$.

Handy, S.L., Cao, X. \& Mokhtarian, P.L. (2006) Self-Selection in the Relationship between the Built Environment and Walking. Journal of the American Planning Association 72 (1), p.55 - 74.

Jakobsson Bergstad, C., Gamble, A., Gärling, T., Hagman, O., Polk, M., Ettema, D., Friman, M., \& Olsson, L. (2011). Subjective well-being related to satisfaction with daily travel. Transportation 38 (1), 1-15.

Johansson, M.V., Heldt, T., \& Johansson, P. (2006). The effects of attitudes and personality traits on mode choice. Transportation Research Part A 40 (6), 507-525.

Kitchen, P., Williams, A, \& Chowhan, J. (2011). Walking to work in Canada: health benefits, socioeconomic characteristics and urban-regional variations. BMC Public Health 2011, 11:212. 
Larrañaga, A.M, Betella, H. B. \& Strambi, O. (2013) The influence of built environment and travel attitudes on walking: A case study of Porto Alegre, Brazil. Working paper. Paper submitted to the International Journal of Sustainable Transportation.

Litman, T. (2007). Transportation elasticities: How Price and other factors affect travel behavior. Victoria Transport Policy Institute, 1250 Rudlin Street, Victoria, BC, V8V 3R7, Canada, 59 p.

Löhner, R. (2010). On the modeling of pedestrian motion. Applied Mathematical Modelling 34 (2), 366-382.

Manaugh, K., \& El-Geneidy, A. (2011). Validating walkability indices: How do different households respond to the walkability of their neighborhood? Transportation Research Part D 16, 309-315.

Márquez, L., Cantillo, V. \& Arellana, J. (2014). How are confort and safety perceived by inland waterway transport passengers? Transport Policy $3646-52$.

Ministerio de Transporte de Colombia (1998). Manual para estudios de origen $y$ destino de transporte de pasajeros y mixto en áreas municipales distritales y metropolitanas, adoptado mediante Decreto No. 1558 del 4 de agosto de 1998.

Mokhtarian, P. \& Cao, X. (2008) Examining the impacts of residential self-selection on travel behavior: A focus on methodologies. Transportation Research B 42(3), 204-228.

Ortúzar, J. D., \& Willumsen, L. G. (2011). Modelling transport. 3a ed. Chichester: John Wiley \& Sons.

Pitombo, C.S., Kawamoto, E., \& Sousa, A.J. (2011). An exploratory analysis of relationships between socioeconomic, land use, activity participation variables and travel patterns. Transport Policy 18, 347-357.

Schwanen, T., Dijst, M., \& Dieleman, F.M. (2002). A microlevel analysis of residential context and travel time. Environment and Planning A 34 (8), 1487-1507.

Train, K. (2003). Discrete Choice Methods with Simulation. Printed in the United Kingdom at the University Press, Cambridge. 329 p. 